\title{
Redescription of Cercopithifilaria bainae Almeida \& Vicente, 1984 (Spirurida, Onchocercidae) from a dog in Sardinia, Italy
}

Domenico Otranto ${ }^{1 *}$, Antonio Varcasia ${ }^{2}$, Cinzia Solinas ${ }^{2}$, Antonio Scala ${ }^{2}$, Emanuele Brianti ${ }^{3}$, Filipe Dantas-Torres ${ }^{1,4}$, Giada Annoscia ${ }^{1}$, Coralie Martin ${ }^{5}$, Yasen Mutafchiev ${ }^{6}$ and Odile Bain ${ }^{5^{\wedge}}$

\begin{abstract}
Background: Three species of the genus Cercopithifilaria have been morphologically and molecularly characterized in dog populations in southern Europe: Cercopithifilaria grassii (Noè, 1907), Cercopithifilaria sp. sensu Otranto et al., 2011 (reported as Cercopithifilaria sp. I), and Cercopithifilaria sp. II sensu Otranto et al., 2012. The adults of Cercopithifilaria sp. I have remained unknown until the present study.

Methods: The material originated from a dog from Sardinia (Italy) diagnosed with dermal microfilariae of Cercopithifilaria sp. I. The holotype and three paratypes of Cercopithifilaria bainae Almeida \& Vicente, 1984, described from dogs in Brazil, were studied as comparative material. A cox1 ( 689 bp) and 12S ( 330 bp) gene fragments were amplified and phylogenetic analysis carried out.

Results: The highest numbers of adult nematodes (82\%) were collected in the sediment of the subcutaneous tissues of the trunk $(n=37)$ and forelimbs $(n=36)$. The morphology of the adult nematodes and microfilariae collected from the dog in Sardinia corresponded to those of C. bainae. All cox 1 and $12 S$ gene sequences showed a high homology (99-100\%) with sequences from microfilariae of Cercopithifilaria sp. I.

Conclusions: The morphological and molecular identity of the microfilariae of C. bainae overlap those described previously as Cercopithifilaria sp. sensu Otranto et al., 2011 (=Cercopithifilaria sp. I). Therefore, the present study reports the occurrence of $C$. bainae in Europe, for the first time after its description and the single record in Brazil. C. bainae appears to be highly diffused in dog populations in southern Europe. The phylogenetic analyses based on cox 1 and 125 do not reveal the three species of Cercopithifilaria parasitizing dogs as a monophyletic group, which suggests that they have derived independently by host switching.
\end{abstract}

Keywords: Canine filarioids, Cercopithifilaria sp. I, Cercopithifilaria sp. II, Cercopithifilaria bainae, Cercopithifilaria grassii, Taxonomy, Europe

\section{Background}

Among the most studied parasites of dogs, Dirofilaria immitis (Leidy, 1856) and Dirofilaria repens (Railliet \& Henry, 1911) (Spirurida, Onchocercidae), causing cardiopulmonary and subcutaneous filariosis, respectively, are characterized by blood circulating microfilariae and are regarded as agents of zoonoses [1]. Aside from these

\footnotetext{
* Correspondence: domenico.otranto@uniba.it

'Deceased

'Dipartimento di Medicina Veterinaria, Università degli Studi di Bari,

Valenzano, BA, Italy

Full list of author information is available at the end of the article
}

two filarial worms, dogs may be parasitized by other less known species (e.g., Onchocerca lupi Rodonaja, 1967 and Cercopithifilaria spp.), whose larvae reside in subcutaneous tissues. In particular, recent studies on filarioids infesting dogs in Europe revealed that they are infected by at least three species of the genus Cercopithifilaria Eberhard, 1980 (see Ref. [2]). Cercopithifilaria grassii (Noè, 1907) was described in the subcutaneous tissue of a dog in Rome (Italy) and was characterized by long $(650 \mu \mathrm{m})$ microfilariae [3]. This species remained neglected until two occasional reports in ticks from dogs in Switzerland [4] and in northern Italy [5]. In the

\section{Biomed Central}


meantime, C. grassii was reported from a dog in Rio de Janeiro, Brazil [6]. Two years later, the same authors, following a careful examination of the material above, described Cercopithifilaria bainae Almeida \& Vicente, 1984, which was distinguished from C. grassii by short (about $180 \mu \mathrm{m}$ ) microfilariae [7]. Recently, microfilariae of Cercopithifilaria sp. sensu Otranto et al., 2011 (further reported as Cercopithifilaria sp. I), were described from a dog in Sicily, Italy [8]. They correspond well to those of $C$. bainae by their body length (mean length of $186.7 \mu \mathrm{m})$. Subsequently, microfilarial infestations by Cercopithifilaria sp. I were reported from dog populations in Spain, Greece and southern Italy, with prevalence up to $21.6 \%$ [9]. In the meantime, a study demonstrated that the microfilariae of Cercopithifilaria sp. I completed their development to the infective stage after two months in Rhipicephalus sanguineus ticks [10]. The microfilariae of C. grassii and Cercopithifilaria sp. II sensu Otranto et al., 2012 were morphologically and molecularly characterized on the basis of specimens from dog population in Europe [2]. However, adult stages of Cercopithifilaria sp. I and II, occurring in dogs in Europe have remained undescribed until the present study.

At the necropsy of a dog positive for microfilariae of Cercopithifilaria sp. I, adult nematodes of Cercopithifilaria sp. were retrieved and used as a basis for the first description of the adults of this parasite occurring in dogs from Europe. In this paper, we report the results of the morphological examination of adult filarial worms corresponding to Cercopithifilaria sp. I and compare them with the type series of $C$. bainae from dogs in Brazil. We demonstrate that the two compared samples are conspecific, and propose the identification of the described European materials as $C$. bainae, thus reporting for the first time this species in Europe. Morphological analysis corroborates with molecular and phylogenetic studies of species of Cercopithifilaria infesting dogs.

\section{Methods}

\section{Collection procedures and morphological study}

The material originated from an 8-year-old male dog from Aglientu (Sardinia, Italy) with a history of tick infestation. The animal was diagnosed positive for dermal microfilariae of Cercopithifilaria sp. I by their morphological observation, according to procedures described elsewhere [8]. Briefly, dermal microfilariae were diagnosed by soaking skin snips in saline solution for $10 \mathrm{~min}$ at $37^{\circ} \mathrm{C}$. A few drops of the sediment were observed under light microscopy (100x) after adding a drop of methylene blue (1\%) and identified [8]. Three months later (October 2012), the same dog was euthanized, according to owner's request due to a rhabdomyosarcoma and associated severe clinical conditions.
One day before euthanasia, the presence of microfilariae of Cercopithifilaria sp. I was confirmed.

Soon after euthanasia, the dog was shaved and necropsy performed. The skin portions at the head, trunk, forelimbs and hind limbs as well as the peri-renal tissue were examined under the stereomicroscope for the presence of parasites. The skin pieces and the carcass were immersed in separate plastic containers in warm $\left(35-39^{\circ} \mathrm{C}\right)$ saline solution for 30 minutes. After removal, four decantations were performed (15 minutes each) in smaller receptacles, until a total volume of $20 \mathrm{ml}$ of sediment was obtained. The number and distribution of the adult nematodes and microfilariae recorded in the sediments are presented in Table 1. Worms were washed in saline solution and transferred into $70 \%$ ethanol.

Microfilariae were collected from the sediment of skin samples before and after the dog was euthanized. In addition, microfilariae were also obtained for comparison from the uterus of one female nematode.

For light-microscopy observations, specimens were cleared and examined as temporary mounts in glycerine. Drawings were made with an optical microscope (Leica Microsystems DMLB 2) equipped with a camera lucida (Leica Microsystems L 3/20). Microscopic images and measures were taken using a digital image processing system (AxioVision rel. 4.8, Carl Zeiss, Germany). Measurement data are given as the range, with the mean $\pm \mathrm{SD}$ in parentheses.

As comparative materials, one male (no. 32.176c, paratype) and one female (no. 32.176d, paratype) of C. bainae were loaned from the Helminthological Collection of the Instituto Oswaldo Cruz (CHIOC), Brazil. Few microfilariae were isolated from the female paratype (no. 32.176d) after permission by Dr. M. Knoff, curator of CHIOC. These specimens were studied in February 2012 by one of the authors (O.B.) who also prepared drawings. In addition, photographs of the holotype (male, no. 32.176a) and a paratype (female, no. 32.176b) were kindly provided by Dr. M. Knoff.

Table 1 Number of adult nematodes (males and females) and microfilariae collected at the necropsy from different anatomical regions

\begin{tabular}{llll}
\hline & Male & Female & Microfilariae \\
\hline Head & 1 & 1 & 25 \\
Fore limbs & 20 & 16 & 2 \\
Hind limbs & - & 3 & 13 \\
Trunk & 11 & 26 & 10 \\
Peri-renal tissue & - & 3 & - \\
Total* $^{*}$ & 38 & 51 & 50 \\
\hline
\end{tabular}

*Total number of adults includes eight nematodes (six males and two females, found after soaking the skinned carcass). 


\section{Molecular amplification and phylogenetic analyses}

Microfilariae $(\mathrm{n}=5)$ and adult nematodes $(\mathrm{n}=8 ; 4$ females and 4 males) were isolated, genomic DNA was extracted using a commercial kit (DNeasy Blood \& Tissue Kit, Qiagen, GmbH, Hilden, Germany). A $c o x 1$ ( 689 bp) and 12S ( 330 bp) gene fragment, were amplified as previously reported [8] and the phylogenetic analysis carried out on a partial cox1 (pcox1; $304 \mathrm{bp}$ ) fragment of Cercopithifilaria spp. [8]. Following purification (Ultrafree-DA columns; Amicon, Millipore; Bedford, USA), amplicons were sequenced directly using the Taq DyeDeoxyTerminator Cycle Sequencing Kit (v.2, Applied Biosystems) in an automated sequencer (ABI-PRISM 377). Sequences were determined from both strands (using the same primers individually as for the PCR) and the electropherograms verified by eye. Following alignment using ClustalW program [11], sequences were compared with those available in GenBank $^{\mathrm{TM}}$ dataset by Basic Local Alignment Search Tool (BLAST - http://blast.ncbi.nlm.nih.gov/Blast.cgi).

To investigate the relationships among filarioids of the Onchocercidae family, sequences of both genes were phylogenetically analysed with those available in GenBank $^{\mathrm{TM}}$. Selected haplotypes of pcox 1 and $12 \mathrm{~S}$ previously sequenced for Cercopithifilaria sp. I (accession numbers: JF461457; JF461461), Cercopithifilaria sp. II (accession numbers: JQ837809, JQ837811) and C. grassii (accession numbers: JQ837810, JQ837812) were included in the analysis. The evolutionary history was carried out using the Neighbour-joining (NJ) method [12] using the Tajima-Nei model [13] and Maximum Likelihood method based on the Kimura 2-parameter model [14]. The evolutionary distances were computed by MEGA5 software [15]. The bootstrap consensus trees inferred from over 8,000 replicates were taken to represent the evolutionary history of the taxa analysed [16]. Dirofilaria immitis was chosen as an out-group (accession numbers: DQ358815). A bootstrap support of 50 was considered significant. The nucleotide sequences analysed in this study are available in the GenBank ${ }^{\mathrm{TM}}$ (accession numbers: KC880117, KC880118).

\section{Results}

All adult nematodes had a subcutaneous localization, with the exception of three gravid females found in the peri-renal adipose tissues. The highest number of adult nematodes $(82 \%)$ was found in the sediment of trunk $(\mathrm{n}=37)$ and forelimb $(\mathrm{n}=36)$ regions, followed by that of posterior limbs and peri-renal tissue (Table 1). Microfilariae were evenly distributed although $50 \%$ of them $(\mathrm{n}=25)$ were found in the sediment from the head. Male $(\mathrm{n}=38)$ and female $(\mathrm{n}=51)$ nematodes were retrieved in all districts examined with a male/female ratio of 0.74 (Table 1 ).
Voucher materials preserved in 70\% ethanol were deposited in the following collection: The Nematode collection of the Muséum National d'Histoire Naturelle, Paris accession number 12YT ( 2 males and 3 females), and Helminthological Collection of the Instituto Oswaldo Cruz accession number CHIOC 35866 (2 males and 3 females) and US National Parasite Collection accession number USNPC 106944.00 ( 2 males and 3 females).

\section{Redescription of Cercopithifilaria bainae (Figures 1, 2, 3 and 4) \\ General}

Slender and delicate onchocercid nematodes (Figure 1A, $1 B$ and $1 C$ ). Anterior end conical, bearing four external labial and four cephalic submedian papillae (forming two laterally elongated rectangles) and a pair of amphids (Figures 1D, 1E and 3A). Oral opening small and round. Buccal capsule in form of flattened ring (Figure 1E). Oesophagus not divided into muscular and glandular portions but with numerous glandular cells in posterior part (Figure 1A and 1B); oesophageal lumen dorsoventrally flattened. Nerve ring at level of anterior and middle third of oesophagus length. Excretory pore not observed. Cuticle thin and smooth. Tail curved ventrally (Figures 2E, K, 3C and 4B) with two later and one longer dorsal conical cuticular processes (lappets) at tail tip; phasmids at base of lateral lappets (Figure 2F).

\section{Male ( $n=7$, see Table 2 for other measurements)}

Body with three or four slight body swellings at $181 \mu \mathrm{m}$ (posterior to nerve ring), at $882 \mu \mathrm{m}$ (posterior to testis reflection) and 2,765 $\mu \mathrm{m}$ (formed by pseudocoelomocyte) in specimens $11.99 \mathrm{~mm}$ long. Body width at level of oesophago-intestinal junction $43-93 \mu \mathrm{m}(58.9 \pm 14.1)$, and at level of cloaca $38.7-46.3 \mu \mathrm{m}(43.0 \pm 3.4)$. Buccal capsule 1.5-2.4 $\mu \mathrm{m}(2.0 \pm 0.6)$ high, 6.7-7.0 $\mu \mathrm{m}(6.9 \pm 0.2)$ wide. Muscular oesophagus 18.7-25.4 $\mu \mathrm{m}(22.6 \pm 2.2)$ wide. Posterior body end forming 3 spiral coils (Figures 2A and 3B). Area rugosa 419.9-575.6 $\mu \mathrm{m}$ (466.6 \pm 63$)$ long, extending in anterior direction from level at 244.5-384.0 $\mu \mathrm{m}(284.8 \pm$ 56.5 ) anterior from cloaca (Figure 2A); consisting of about 80 similar in size ventral transverse bands; each band bearing a line of fine longitudinal striations (Figure 2B). Testis reflection at 401.0-780.3 $\mu \mathrm{m}(592.2 \pm 116.1)$, from anterior body end (Figure 1A). Caudal papillae presented by single ventral median precloacal papilla and from 5 to 6 pairs of subventral papillae (Figure 2C, 2D and 2E). Subventral pairs of papillae situated as follow: two or three pairs at level of cloaca, two pairs just posterior to cloaca, one pair of papillae at mid-tail length (usually absent or presented by a single papilla) and one or two pairs of subterminal papillae (full set of caudal papillae was never observed). Right spicule with conspicuous dorsal heel (Figure 2G). Left spicule with hook at distal end 
Figure 1 Cercopithifilaria bainae. A) Anterior part, male, lateral view; note testis reflection (arrow). B-E) Female morphology. B) Anterior part, lateral view, note the body swellings posterior of nerve ring and at the level of vagina. C) Anterior part with body swellings (arrows), lateral view. D) Anterior end, apical view; E) Anterior end lateral and dorsoventral views, respectively. Scale-bars in micrometers.

(Figure 2D); length of lamina/length of handle ratio 0.50.7 (Figure 2H). Length of left spicule/length of right spicule ratio 3.9-4.3.

\section{Female ( $n=7$, see Table 2 for other measurements)}

Body with four slight body swellings at $203 \mu \mathrm{m}$ (posterior to nerve ring), at $485 \mu \mathrm{m}$ (at level of vagina), at 1,618 $\mu \mathrm{m}$ (formed by pseudocoelomocyte) and at 2,206 $\mu \mathrm{m}$ (formed by pseudocoelomocyte), in a specimen $17.25 \mathrm{~mm}$ long (Figure 1B and 1C). Body width at level of oesophagointestinal junction $78-120 \mu \mathrm{m}(96.1 \pm 11)$; body width at anus 35.4-42.0 $\mu \mathrm{m}$ (38.6 \pm 3$)$. Buccal capsule 3.0-3.2 $\mu \mathrm{m}$ (3.1 \pm 0.1$)$ high, 15.9-16.8 $\mu \mathrm{m}(16.4 \pm 0.6)$ wide. Oesophagus 24.7-30.0 $\mu \mathrm{m} \quad(27.6 \pm 26) \quad$ wide. Reproductive system didelphic opistodelphic. Vulva slit like. Vagina muscular 57.5 - $69.7 \mu \mathrm{m}(63.4 \pm 6.1)$ long, 32.8-35.3 $\mu \mathrm{m}(34.4 \pm 1.4)$ wide (Figures 2I, J and 4A). Ovijector about 1,000 $\mu \mathrm{m}$ long, posteriorly directed, with circular muscular walls (Figures 1B and 2I). Tail long and slender, bent ventrally (Figure 2K).

\section{Microfilariae (see Table 3 for measurements; Figure 4C)}

Skin microfilariae unsheated. Cephalic end rounded with slight protuberance bearing a tiny cephalic hook. Body short, flattened dorso-ventrally. Body width constant along its length, except for the posterior conical end (about $30 \mu \mathrm{m}$ long). Tail pointed. Body cuticle thick bearing transverse striations. Live microfilariae presented slow caudal movements.

\section{Remarks}

The morphology of C. bainae has been known only from its brief original record in Brazil [7]. For the adequate identification of the newly collected material from Sardinia, we had the opportunity to obtain additional information from the holotype and three paratypes of C. bainae. The photos of the holotype (male, no. 32.176a) demonstrate characters corresponding to the morphology of the genus Cercopithifilaria. These are an undivided oesophagus $365 \mu \mathrm{m}$ long, a posterior body end forming 3 spiral coils and an area rugosa $586 \mu \mathrm{m}$ long extending in anterior direction from level at $345 \mu \mathrm{m}$ from the cloaca (Figure $5 \mathrm{~A}$ and 5B). The female (paratype no. 32.176d) possesses characters corresponding to the generic diagnosis of Cercopithifilaria, i.e. an undivided oesophagus $470 \mu \mathrm{m}$ long, a vulva situated at $530 \mu \mathrm{m}$ from the anterior body end (Figure 6A) and a tail with three lappets (Figure 6B 


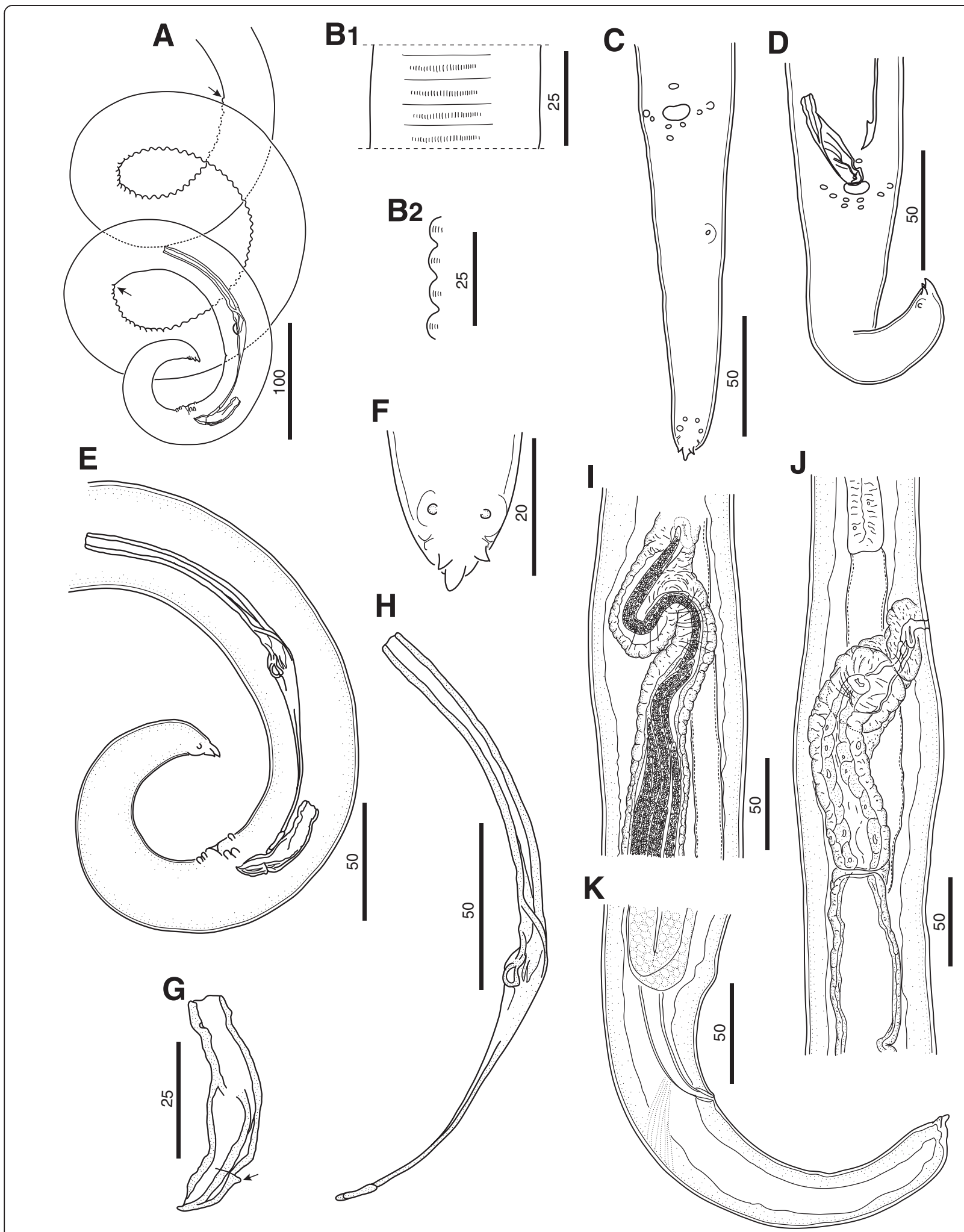

Figure 2 Cercopithifilaria bainae. A-H) Male morphology. A) Posterior part, male, lateral view; note ends of area rugosa (arrows). B) Area rugosa, detail, ventral (B1) and lateral view (B2), respectively. C) Tail, ventral view. D) Tail; note the hook-like distal end of left spicule in ventral view. E) Posterior end, lateral view. F) Tail extremity, ventral view. G) Right spicule, sinistral view, note the dorsal heel; (arrow). H) Left spicule, sinistral view. I-K) Female morphology. I) Vagina, ventral view; note microfilariae in the vagina end ovijector. J) Vagina, lateral view; note the oesophago-intestinal junction. K) Posterior end, lateral view. Scale-bars in micrometers. 


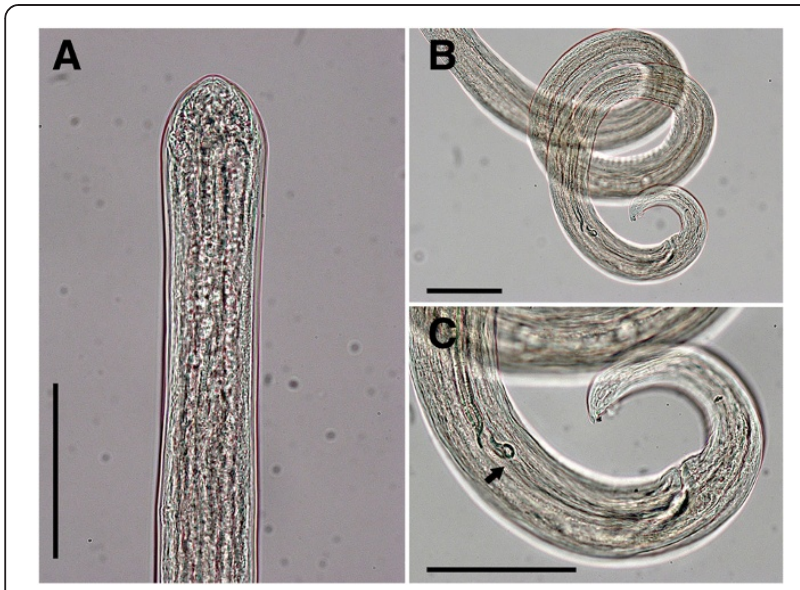

Figure 3 Cercopithifilaria bainae. Light-microscopy. A-C) Male morphology, lateral view. A) Anterior end. B) Spirally coiled posterior body end. C) Posterior end with spicules; note junction of lamina and handle (arrow).

and $6 \mathrm{C}$ ). The photos of the second female (paratype no. $32.176 \mathrm{~b})$ exhibited morphology corresponding to that of the female no. 32.176d. However, the second male specimen (paratype no. 32.176c) is characterized by an oesophagus divided into muscular and glandular portions $(2,120 \mu \mathrm{m}$ long), right spicule $50 \mu \mathrm{m}$ long, left spicule $230 \mu \mathrm{m}$ long and three pairs of regularly arranged precloacal papillae (Figure 6D). The characters of the latter male individual corresponds to the morphology of Acanthocheilonema reconditum (Grassi, 1889) as redescribed by Korkejian and Edeson, 1978 [17]. Thus, the four studied type specimens reveal that the original description of this species was based on heterogeneous materials of two species, $C$. bainae (one male and two females, including the holotype) and $A$. reconditum (one male). Both species are characterized by similar body dimensions, spicule length, position of vulva and length of

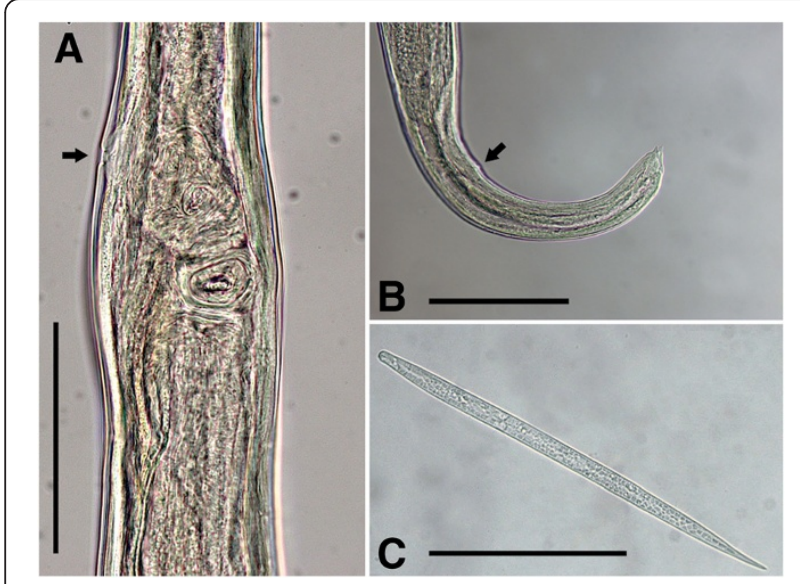

Figure 4 Cercopithifilaria bainae. Light-microscopy. A-B) Female morphology, lateral view. A) Vagina; note vulva (arrow). B) Tail; note anus (arrow). C) Microfilaria dorso-ventral view. Scale-bars $=100 \mu \mathrm{m}$. tail (Table 2), but can be differentiated by the morphology of the oesophagus, the arrangement of the caudal papillae and the area rugosa in males. During the survey of the nematode parasitizing dogs in Brazil, when C. bainae were collected, $43.1 \%$ of the dogs were positive for A. reconditum [6,7]. Based on the discussion above, we conclude that the reported long glandular oesophagus in the original description of C. bainae [7] is an artefact resulting from the presence of $A$. reconditum in the type series.

The morphology of the adult nematodes and microfilariae collected from the skin snip and the uteri of the female worm from the dog in Sardinia correspond well with those of $C$. bainae (Tables 2 and 3). Therefore, we regard the samples described in the present study as conspecific with $C$. bainae.

\section{Molecular analyses}

All cox 1 and $12 \mathrm{~S}$ gene sequences from adults and microfilariae from the studied dog are characterized by 99 and $100 \%$ homology, respectively. BLAST analysis of cox 1 and $12 \mathrm{~S}$ sequences from the male and female individuals examined showed a high homology (99-100\%) with sequences from microfilariae of Cercopithifilaria sp. I (cox1: JF461457; 12S: JF461461, respectively). In particular, cox 1 haplotype I ( $\mathrm{HI} ; \mathrm{n}=6)$, and two new haplotypes (HXVII and HXVIII), differing for a single polymorphism from HI, were detected (accession numbers: KC880117, KC880118). For both genes, the phylogenetic analyses of Cercopithifilaria sp. here examined with those of all other Cercopithifilaria species available in GenBank were concordant in clustering their sequences with the others in the genus, including those available from dogs (i.e., Cercopithifilaria sp. I, Cercopithifilaria sp. II and C. grassii) (Figures 7 and 8). In addition, the analysis showed that sequences of Cercopithifilaria here examined clustered with those of Cercopithifilaria sp. I (bootstrap value, 99\%). The branches were supported by high bootstrap values in their main nodal points. There was consistency in the topology of the tree inferred by the NJ and ML (not shown) methods (for both target genes). The phylogenetic trees based on regions of $12 \mathrm{~S}$ and $\operatorname{cox} 1$ sequence data are presented in Figures 7 and 8 , respectively.

\section{Discussion}

The present study reports, for the first time, C. bainae after its description in Brazil [7]. The morphological and molecular identity of the microfilariae of $C$. bainae with those described previously as Cercopithifilaria sp. sensu Otranto et al., 2011 [2,9,10,18-20] and Cercopithifilaria sp. I [1] from dog populations in Europe indicated that these studies were dealing with $C$. bainae. Therefore, these data suggest that $C$. bainae is widely diffused in 
Table 2 Measurements of adults of Cercopithifilaria spp. reported from dogs

\begin{tabular}{|c|c|c|c|}
\hline Species & C. bainae & C. bainae & C. grassii \\
\hline Country & Sardinia (Italy) & Brazil & Italy \\
\hline Ref. & present study & Almeida and Vicente, 1984 & Noè, 1911 \\
\hline Female & $(n=23)$ & - & - \\
\hline Body length (mm) & $14.0-19.0(16.1 \pm 1.3)$ & $13.6-17.9$ & $17.0-21.0$ \\
\hline Nerve ring, from anterior extremity & $170-209(185.8 \pm 11.2)$ & $140-180$ & 215 \\
\hline Oesophagus, length & $393-541(467.7 \pm 37.2)$ & $380-400$ & - \\
\hline Vulva, from anterior extremity & $412-598(479.8 \pm 48.9)$ & $430-540$ & 600 \\
\hline Tail, length & $148-246(199.8 \pm 35.1)$ & $120-170$ & 220 \\
\hline Male & $(n=13)$ & - & - \\
\hline Body length (mm) & $9.0-12.0(10.7 \pm 1.1)$ & 7.3-9.1 & $7.0-8.0$ \\
\hline Nerve ring, from anterior extremity & $144-187(172.6 \pm 15.8)$ & $110-120$ & - \\
\hline Oesophagus, length & $358-493(449.8 \pm 72.3)$ & $380-400$ & - \\
\hline Left spicule, length & $184-205(196.5 \pm 9.2)$ & $180-240$ & - \\
\hline Right spicule, length & $43-52(46.9 \pm 3.6)$ & $46-82$ & - \\
\hline Tail, length & $111-151(129.8 \pm 14.3)$ & - & - \\
\hline
\end{tabular}

Measurements are in micrometers unless otherwise specified.

European dog populations. The phylogenetic topology inferred by $12 \mathrm{~S}$ and cox 1 (data not shown) of the adult specimens here studied with the sequences of Cercopithifilaria sp. I clearly show a close homology between them. Indeed, the nucleotide homology supports the morphological diagnosis above. The retrieval of haplotype I in six specimens confirms previous data that this haplotype is the most represented in the nematode populations from the Mediterranean basin [19], including Sardinia. However, based on the retrieval of two further haplotypes (i.e., HXVII and HXVIII), the high genetic variability in the Cercopithifilaria sp. I population is further assessed. The number of haplotypes detected in the nematodes collected from the same animal $(n=3)$ and the mean value of nucleotide variability between them, were lower than those previously recorded (i.e., 14 haplotypes and mean variability $1.6 \%$ ), within populations of Cercopithifilaria sp. I microfilariae collected from dogs and ticks from different geographical areas (i.e., Italy, Spain and Greece) of the Mediterranean basin [19].

Almeida and Vicente [6,7] established that $19.5 \%$ of the dogs studied in Rio de Janeiro were positive for C. bainae. This number falls within the prevalence range (4.3-21.6\%) of microfilariae morphologically identical to those of C. bainae reported from dog populations of southern Europe [9]. The data of the distribution and the high prevalence of $C$. bainae indicate that this species is probably a common parasite of dogs at

Table 3 Measurements (in micrometers) and morphological features of dermal microfilariae of Cercopithifilaria spp. reported from dogs

\begin{tabular}{|c|c|c|c|c|}
\hline Species & Cercopithifilaria sp. I & C. bainae & C. grassii & Cercopithifilaria sp. II \\
\hline Ref. & Otranto et al., 2011 & Almeida and Vicente, 1984 & Noè, 1911 & Otranto et al., 2012 \\
\hline & $(n=185)$ & - & - & $(n=6)$ \\
\hline Body, length & $170-197$ & 185 & $635-670$ & $273-305$ \\
\hline Body width, dorso-ventral view & $6.1-9.4$ & 6.6 & $15-17$ & $12-15^{*}$ \\
\hline Body width, lateral view & $3-3.5$ & n.r. & $15-17$ & $9-10.5$ \\
\hline Lateral alae & no & n.r. & no & well developed \\
\hline Anterior extremity & slightly attenuated & slightly attenuated & bulbous & without alae and thinner \\
\hline Cuticular posterior end, length & 15 & n.r. & $18-28$ & $38-48$ \\
\hline Shape of caudal extremity & blunt & blunt & bifid & shortly attenuated \\
\hline Internal anatomy & nuclei prominent & n.r. & nuclei not visible & nuclei prominent \\
\hline
\end{tabular}




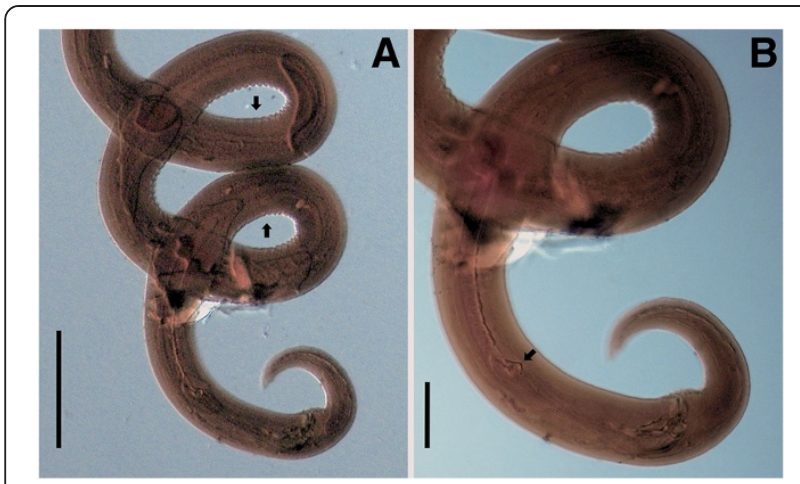

Figure 5 Cercopithifilaria bainae, type specimens, light-microscopy (photos were obtained by Dr. M. Knoff). A-B) Holotype, male (CHIOC no. 32.176a). A) Spirally coiled posterior body end. B) Posterior; note junction of lamina and handle (arrow).

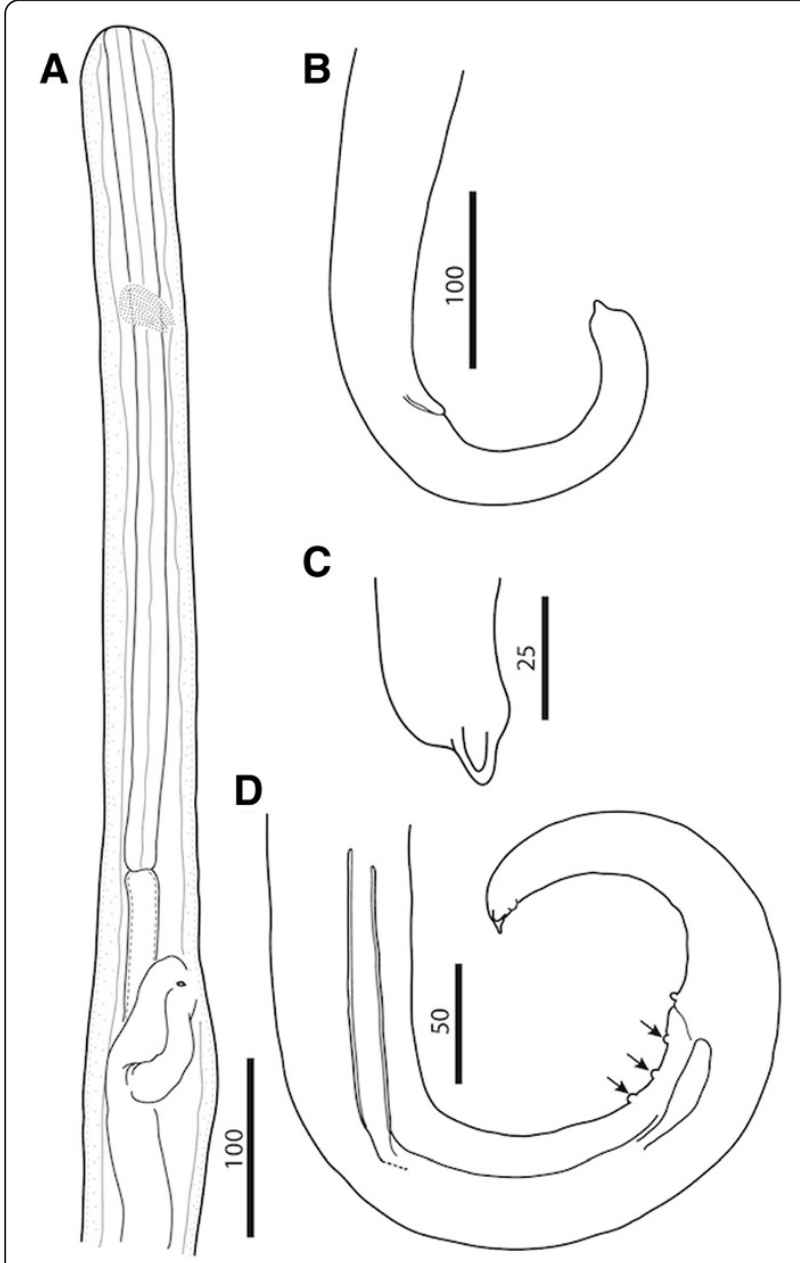

Figure 6 A-C) Cercopithifilaria bainae, paratype, female (CHIOC no. 32.176d). A) Anterior end, ventro-lateral view. B) Posterior end, lateral view. C) Tail extremity, lateral view. D) Acanthoceilonema reconditum, male from the type series of C. bainae (CHIOC no. 32.176c), posterior end, lateral view; note precloacal papillae (arrows). Scale bars in micrometers. least in the subtropical and tropical regions of the southern and northern hemispheres. This finding is not surprising if one considers that $R$. sanguineus, regarded as the vector of this filarioid [10] is distributed in vast geographical areas as a consequence of its adaptability to different climatic conditions and of its close association with the domestic dog [21]. In addition, the high prevalence of infestation in ticks collected from different European countries [9] and the finding of up to 1,469 developing larvae in a single tick specimen suggest that Cercopithifilaria sp. I infestation is well tolerated by $R$. sanguineus, which could explain the broad distribution of this nematode among tick-infested dogs. Whether different populations of $R$. sanguineus have influenced the speciation of these nematodes has yet to be investigated. The wide geographical distribution of C. bainae among dogs from urban, wooded and rural Mediterranean areas [9], the well structured genetic population with up to 19 haplotypes identified so far (Ref. 19 and present study) indicates the role of tick vectors in the transmission of different strains/populations and in the speciation of these three species of Cercopithifilaria. Without a doubt, the definition of the species here studied clearly provides new data instrumental to the scientific debate on the evolution and on the high degree of diversity among taxa within this genus.

Based on the morphological characters, the host range and the geographical distribution, several studies have suggested that the ancestral hosts of Cercopithifilaria were most probably ruminants of the families Bovidae and Cervidae [22-24]. The reduced number of caudal papillae (6 or 7 instead of 9 pairs) in males, the compact arrangement of the precloacal papillae around cloaca (in contrast to the equidistant and aligned papillae), together with the reduced number of cephalic papillae (6 instead of 8) and reduced caudal lappets (from three to two lappets, or entire reduction to a pointed terminal end) are considered as derived characters observed mainly in species parasitizing hosts distinct from ruminants, such as baboons (Cercopithecidae), civets (Nandiniidae), porcupines (Hystricidae), etc. (summarized in Ref. 22). However, a molecular study based on $\operatorname{cox} 1$ gene of 7 species of parasites of Japanese cervids suggests that these characters, considered apomorphic, could derive independently [25]. The present study reveals that the males of $C$. bainae possess only six pairs of caudal papillae, similar to those of Cercopithifilaria kenyensis (Eberhard, 1980), Cercopithifilaria eberhardi Bain, Wamae \& Reid, 1988 and Cercopithifilaria narokensis Bain, Wamae \& Reid, 1988 (all three parasites of baboons), as described in Refs. [26,27]. However, the arrangement of the first two pairs of postcloacal papillae of C. bainae is distinct as well as the shape of the terminal caudal end. The description of $C$. grassii does not reveal in detail the 


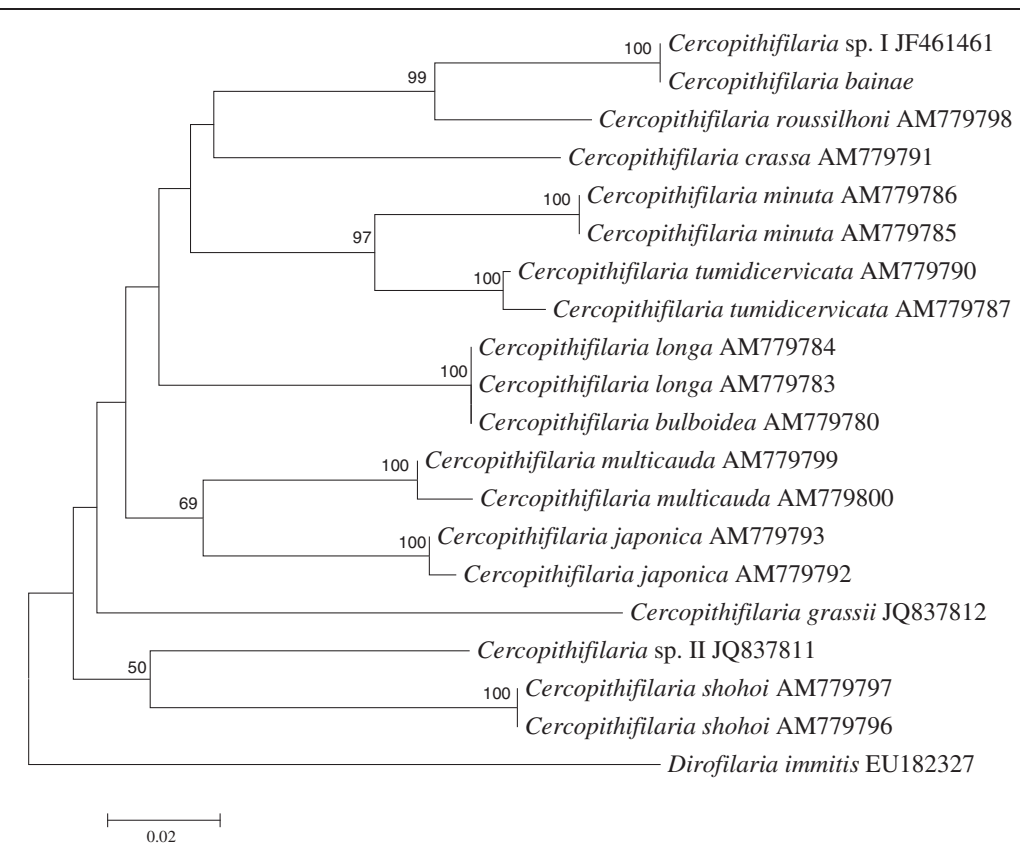

Figure 7 Phylogenetic tree based on $12 \mathrm{~S}$ sequence data, compared with those representatives of the genus Cercopithifilaria available in GenBank ${ }^{\mathrm{TM}}$. The tree was constructed using Neighbor-Joining (NJ) method and rooted against Dirofilaria immitis out-group.

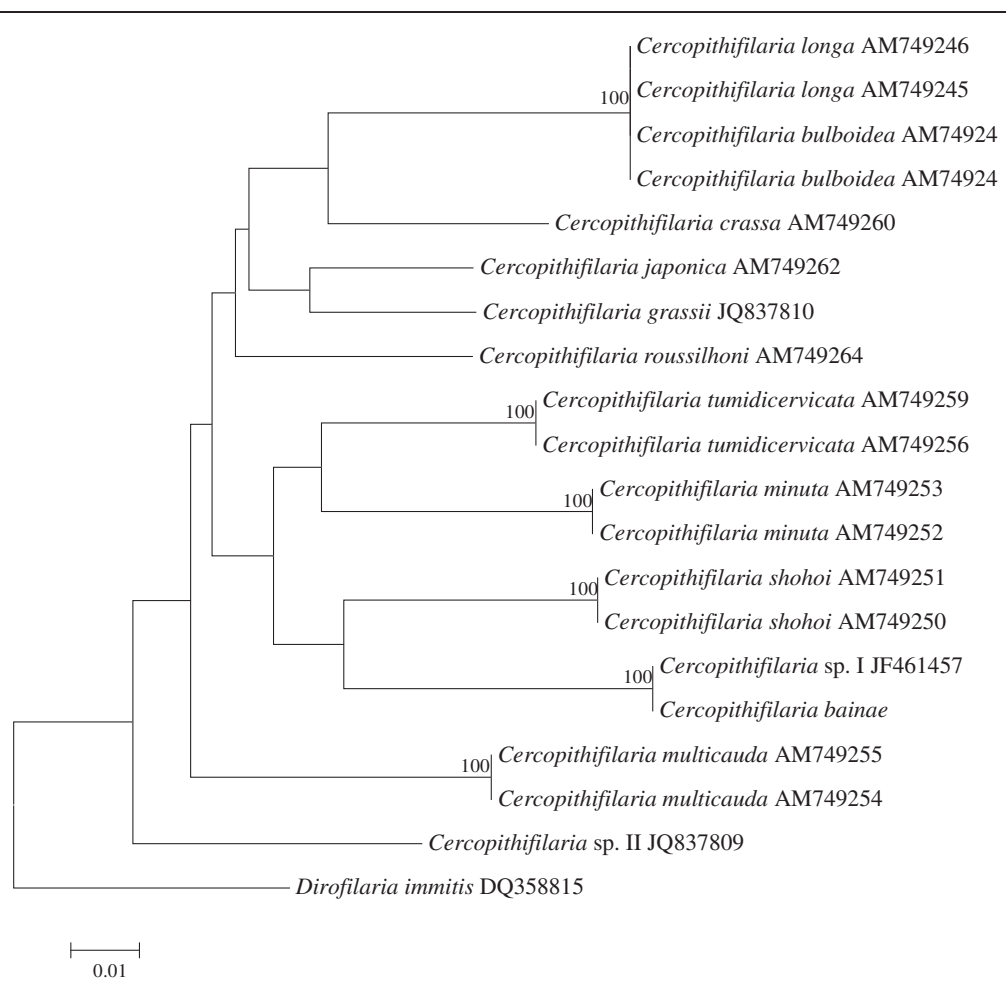

Figure 8 Phylogenetic tree based on cox 1 sequence data, compared with those representatives of the genus Cercopithifilaria available in GenBank ${ }^{\mathrm{TM}}$. The tree was constructed using Neighbor-Joining (NJ) method and rooted against Dirofilaria immitis out-group. 
number and the arrangement of the caudal papillae, while the adults of Cercopithifilaria sp. II are still unknown, therefore, we cannot compare these characters with those of C. bainae.

The present phylogenetic analyses are based on 12 Cercopithifilaria spp., i.e. C. shohoi Uni, Suzuki \& Katsumi, 1998, C. multicauda Uni \& Bain, 2001, C. minuta Uni \& Bain, 2001, C. tumidicervicata Uni \& Bain, 2001 and C. bulboidea Uni \& Bain, 2001 parasitic in Bovidae in Japan [23,28], C. longa Uni, Bain \& Takaoka, 2002 and C. crassa Uni, Bain \& Takaoka, 2002 from Cervidae in Japan (Uni et al. 2002), C. japonica (Uni, 1983) from Ursidae in Japan [29], C. roussilhoni Bain, Petit \& Chabaud, 1986 from Hystricidae in Gabon (Africa) [30] and C. grassii, C. bainae and Cercopithifilaria sp. II from dogs (Canidae) in Europe [2]. The phylogenetic trees of cox 1 and $12 S$ genes differ from one another by their topologies. However, in both analyses, the species from dogs were not grouped in a monophyletic group, which could suggest that they have derived independently by host switching. At present, the phylogenetic studies within Cercopithifilaria are based mainly on species from Japan. Therefore, further studies involving more species from various geographical regions could provide more accurate phylogenetic hypothesis for this group. Unfortunately, there is no reliable information about the morphology of the adults of C. grassii, while those of Cercopithifilaria sp. II are still unknown.

\section{Conclusion}

Filarioids localizing in the subcutaneous tissues of dogs (e.g., Cercopithifilaria spp. as well as O. lupi) remain almost unknown to the majority of the parasitologists and veterinarians for the dermal localization of the adult and microfilariae and thus due to the difficulties in collecting skin snips for their detection. Again, while O. lupi is of increasing zoonotic interest and it also causes severe ocular disease of dogs [1], only a few clinical and histological alterations have been recorded in the course of Cercopithifilaria sp. infestation [18]. Nonetheless, information here reported clearly place $C$. bainae among the parasites that would deserve to be better studied in many aspects, such as the antigen interactions with the host immune system and the insurgence of atypical dermatitis in affected animals.

\section{Competing interest}

The authors declare that they have no competing interest.

\section{Authors' contributions}

$\mathrm{DO}, \mathrm{OB}, \mathrm{EB}, \mathrm{AV}, \mathrm{FD}-\mathrm{T}$ and $\mathrm{YM}$ conceived the research, wrote the first draft, contributed with data analysis and interpretation, and revised the manuscript. AV, CS collected samples. YM and OB described parasites and performed drawings and share the senior authorship. GA ran the molecular assays. CM, AS contributed with interpretation and revision of the manuscript. Due to the sudden death of $O B$ she did not read the manuscript whereas all the authors read and approved the final version of the manuscript.

\section{Acknowledgments}

The authors are grateful to Dr. Marcelo Knoff (Curator of the Helmintológica do Instituto Oswaldo Cruz, Laboratório de Helmintos Parasitos de Vertebrados Rio de Janeiro, Brazil) for his great support and kindness in providing paratypes of $C$. bainae for morphological studies and preparation of the digital images of the type material. Authors thank Dr. N. Columbano and Dr. A.P. Pipia for help in the collection of samples. Prof. S. Giannetto is thanked for his critical review of the manuscript. The research was partially funded by a grant of the Regional Government of Sardinia, prot. CRP2 134 (L.R. 7, 2007). Authors wish to thank Merial, France for partially supporting this study.

$\mathrm{DO}, \mathrm{AV}, \mathrm{CS} E \mathrm{~B}, \mathrm{FD}-\mathrm{T}, \mathrm{AS}, \mathrm{GA}, \mathrm{CM}$ and YM dedicate this article to the memory of our friend and colleague Odile Bain who greatly contributed to this article and who died in Paris on the 16th October 2012.

\section{Author details}

${ }^{1}$ Dipartimento di Medicina Veterinaria, Università degli Studi di Bari, Valenzano, BA, Italy. ${ }^{2}$ Dipartimento di Medicina Veterinaria, Università degli Studi di Sassari, Sassari, Italy. ${ }^{3}$ Dipartimento di Scienze Veterinarie, Università degli Studi di Messina, Messina, Italy. ${ }^{4}$ Departamento de Imunologia, Centro de Pesquisas Aggeu Magalhães, Recife, PE, Brazil. ${ }^{5}$ Departement Systématique et Evolution, UMR 7245 CNRS, Muséum National d'Histoire Naturelle, Paris, France. ${ }^{6}$ Institute of Biodiversity and Ecosystem Research, Bulgarian Academy of Sciences, Sofia, Bulgaria.

Received: 25 March 2013 Accepted: 18 April 2013 Published: 4 May 2013

\section{References}

1. Otranto D, Dantas-Torres F, Brianti E, Traversa D, Petri D, Genchi C, Capelli G: Vector-borne helminths of dogs and humans in Europe. Parasit Vectors 2013, 6:16.

2. Otranto D, Brianti E, Dantas-Torres F, Miró G, Latrofa MS, Mutafchiev Y, Bain O: Species diversity of dermal microfilariae of the genus Cercopithifilaria infesting dogs in the Mediterranean region. Parasitology 2012, 140:99-108.

3. Noè G: Contribuzioni alla Sistematica e alla Anatomia del Genere Filaria. La Filaria grassii. Roma: Istituto di Anatomia Comparata della Regia Università di Roma; 1907:236-252

4. Bain $O$, Aeschlimann $A$, Chatelanat P: Présence, chez des tiques de la région de Genève, de larves infestantes qui pourraient se rapporter à la filaire de chien Dipetalonema grassii. Ann Parasitol Hum Comp 1982, 57:643-646.

5. Pampiglione S, Canestri Trotti G, Marchetti S: Ritrovamento di Diptalonema grassii (Noè, 1907) in Rhipicephalus sanguineus su cane in Italia e descrizione di alcuni suoi stadi larvali. Parassitologia 1983, 25:316.

6. Almeida GLG, Vicente JJ: Dipetalonema reconditum (Grassi 1890) Dipetalonema grassii (Noè, 1907) e Dirofilaria immitis (Leidy, 1856) em cães na cidade do Rio de Janeiro (Nematoda - Filarioidea). Atas Soc Biol Rio de Janeiro 1982, 23:9-12.

7. Almeida GLG, Vicente JJ: Cercopithifilaria bainae sp. n. parasita de Canis familiaris (L.) (Nematoda, Filarioidea). Atas Soc Biol Rio de Janeiro 1984, 24:18.

8. Otranto D, Brianti E, Dantas-Torres F, Weigl S, Latrofa MS, Gaglio G, Cauquil L, Giannetto S, Bain O: Morphological and molecular data on the dermal microfilariae of a species of Cercopithifilaria from a dog in Sicily. Vet Parasitol 2011, 182:221-229.

9. Otranto D, Brianti E, Latrofa MS, Annoscia G, Weigl S, Lia RP, Gaglio G, Napoli E, Giannetto S, Papadopoulos E, Mirò G, Dantas-Torres F, Bain O: On a Cercopithifilaria sp. transmitted by Rhipicephalus sanguineus: a neglected, but widespread filarioid of dogs. Parasit Vectors 2012, 5:1.

10. Brianti E, Otranto D, Dantas-Torres F, Weigl S, Latrofa MS, Gaglio G, Napoli E, Brucato G, Cauquil L, Giannetto S, Bain O: Rhipicephalus sanguineus (Ixodida, Ixodidae) as intermediate host of a canine neglected filarial species with dermal microfilariae. Vet Parasitol 2012, 10:330-337.

11. Larkin MA, Blackshields G, Brown NP, Chenna R, McGettigan PA, McWilliam H, Valentin F, Wallace IM, Wilm A, Lopez R, Thompson JD, Gibson TJ, Higgins DG: ClustalW and ClustalX version 2. Bioinformatics 2007, 23:2947-2948.

12. Saitou N, Nei M: The neighbor-joining method: a new method for reconstructing phylogenetic trees. Mol Biol Evol 1987, 4:406-425. 
13. Tajima F, Nei M: Estimation of evolutionary distance between nucleotide sequences. Mol Biol Evol 1984, 1:269-285.

14. Kimura M: A simple method for estimating evolutionary rate of base substitutions through comparative studies of nucleotide sequences. J Mol Evol 1980, 16:111-120.

15. Tamura K, Peterson D, Peterson N, Stecher G, Nei M, Kumar S: MEGA5: molecular evolutionary genetics analysis using maximum likelihood, evolutionary distance, and maximum parsimony methods. Mol Biol Evol 2011, 28:2731-2739.

16. Felsenstein J: Confidence limits on phylogenies: An approach using the bootstrap. Evolution 1985, 39:783-791.

17. Korkejian A, Edeson JF: Studies on naturally occurring filarial infections in dogs in Lebanon. Ann Trop Med Parasitol 1978, 72(1):65-78

18. Otranto D, Brianti E, Abramo F, Gaglio G, Napoli E, Latrofa MS, Ramos RA, Dantas-Torres F, Bain O: Cutaneous distribution and localization of Cercopithifilaria sp. microfilariae in dogs. Vet Parasitol 2012, 190(1-2):143-150.

19. Otranto D, Latrofa MS, Brianti E, Annoscia G, Parisi A, Dantas-Torres F, Bain $\mathrm{O}$, Gasser RB: An assessment of genetic variability in the mitochondrial cytochrome c oxidase subunit 1 gene of Cercopithifilaria sp. (Spirurida, Onchocercidae) from dog and Rhipicephalus sanguineus populations. Mol Cell Probes 2012, 26:81-89.

20. Latrofa MS, Weigl S, Dantas-Torres F, Annoscia G, Traversa D, Brianti E, Otranto D: A multiplex PCR for the simultaneous detection of species of filarioids infesting dogs. Acta Trop 2012, 122(1):150-154.

21. Dantas-Torres F: Biology and ecology of the brown dog tick, Rhipicephalus sanguineus. Parasit Vectors 2010, 3:26.

22. Bain O, Uni S, Takaoka H: A: A synthetic look at a twenty years old taxon, Cercopithifilaria - its probable evolution. In Proceedings of the 10th International Congress of Parasitology (ICOPA). Vancouver (Canada): Monduzzi Editore; 2002:365-368.

23. Uni S, Suzuki Y, Baba M, Mitani N, Takaoka H, Katsumi A, Bain O: Coexistence of five Cercopithifilaria species in the Japanese rupicaprine bovid, Capricornis crispus. Parasite 2001, 8:197-213.

24. Uni S, Bain O, Takaoka H, Fujita H, Suzuki Y: Diversification of Cercopithifilaria species in Japanese wild ruminants with description of two new species. Parasite 2002, 9:293-304.

25. Agatsuma T, Iwagami M, Uni S, Takaoka H, Katsumi A, Kimura E, Bain O: Molecular phylogenetic relationships among seven Japanese species of Cercopithifilaria. Parasitol Int 2005, 54:195-199.

26. Eberhard ML: Dipetalonema (Cercopithifilaria) kenyensis subgen. et sp. $\mathrm{n}$. (Nematoda: Filarioidea) from African baboons, Papio anubis. J Parasitol 1980, 66(3):551-554.

27. Bain O, Wamae CN, Reid GDF: Diversité des Filaires du genre Cercopithifilaria chez les Babouins au Kenya. Ann Parasit Hum Comp 1988, 63:224-239

28. Uni S, Suzuki Y, Katsumi A: Cercopithifilaria shohoi n. sp. (Nematoda: Filarioidea) from the relict Bovidae, Capricornis crispus, in Japan. Parasite 1998, 5(2):119-126

29. Uni S: Filarial parasites from the black bear of Japan. Ann Parasit Hum Comp 1983, 58(1):71-84.

30. Bain O, Petit G, Chabaud AG: Une nouvelle filaire, Cercopithifilaria roussilhoni n. sp., parasite de l'Athérure au Gabon, transmise par tiques: hypothèse sur l'évolution du genre. Ann Parasit Hum Comp 1986, 61:81-93.

\section{Submit your next manuscript to BioMed Central and take full advantage of:}

- Convenient online submission

- Thorough peer review

- No space constraints or color figure charges

- Immediate publication on acceptance

- Inclusion in PubMed, CAS, Scopus and Google Scholar

- Research which is freely available for redistribution 